\title{
Incidental Finding of MEGDEL Syndrome Based on Neuroimaging: Case Report
}

\author{
Salma A. Alshammari Fouad A. Alghamdi Rami Alhazmi \\ Shaikhah Aldossary
}

King Fahad Specialist Hospital, Dammam, Saudi Arabia

\section{Keywords}

Leigh-like syndrome $\cdot$ Dystonia $\cdot$ Sensorineural hearing loss

\begin{abstract}
MEGDEL 3-methylglutaconic (MG) aciduria, deafness, encephalopathy, Leigh-like syndrome is an autosomal recessive disorder associated with infantile hypoglycemia, progressive psychomotor developmental delay, cerebellar atrophy with lesions in the basal ganglia, spasticity, dystonia, deafness, and transient liver problems, which typically occur in the first year of life. Other clinical presentations include failure to thrive, epilepsy, and optic nerve atrophy. The serine active site-containing 1 (SERAC1) mutation is localized at the mitochondria-associated membranes, which are responsible for encoding a phosphatidylglycerol remodeler essential for both mitochondrial function and intracellular cholesterol trafficking and is thus responsible for the disease. Diagnosis is confirmed by the elevation of and concentrations of 3-MG acid and 3-methylglutaric acid in the urine or by identification of bi-allelic SERAC1 pathogenic variants on molecular genetic testing. Different pathological variants of SERAC1 have been identified in MEGDEL syndrome to date. Here, we report a case of a child with MEGDEL syndrome due to SERAC1 mutation. The child presented with accidental finding by CT showing hypodensity on bilateral symmetric anterior putamen and caudate abnormal. Neurological examination was unremarkable. This report presents a new neuroimaging finding by CT of MEGDEL syndrome.
\end{abstract}

\section{Introduction}

MEGDEL syndrome is a rare inborn error of metabolism. Serine active site-containing 1 (SERAC1) mutation has different variants and is associated with several diseases and syndromes, including MEGDEL syndrome. The prevalence, incidence, and pattern of MEGDEL 
syndrome are unknown not just in Saudi Arabia but also worldwide. Hence, knowing the pattern of the disease will help in managing such cases.

\section{Case Report}

A 5-year-old boy with consanguineous Saudi parents was referred from the neurosurgery department to the pediatric neurology clinic. At age of 3 years, he had fallen on the head from a height of $1.5 \mathrm{~m}$ and presented to the emergency department with repeated vomiting but with no loss of consciousness, headache, or seizures. On brain CT scan, a linear fracture was found on the right frontal bone. Incidentally, basal ganglia changes were observed on imaging (Fig. 1). Neurological examination was unremarkable, aside from speech and motor delays. His mother had an uneventful, full-term pregnancy, and he was delivered by cesarean section due to malposition without any complications. He was in the well-baby nursery for 2 weeks because of jaundice. Following are his developmental milestones: he rolled over when he was 5 months old, sat at 8 months, and walked at 20 months of age. He was able to say "mama," "baba," and 3-4 other words, at age of 3 years old. However, he could still not form sentences. A year later, the child started to loss his previously acquired skills, he lost the ability to sitting at age of 4 years and the setting few months later. Currently, he is not able to roll over or to control his neck. Furthermore, he lost the speech and started to have frequent chocking. His mother had a history of 2 first trimester abortions for unknown reasons. The patient had a 14-year-old sister who passed away several months prior - this sibling had generalized developmental delay, spastic quadriplegic cerebral palsy, and epilepsy.

On physical examination, our patient had stable vital signs and had no dysmorphic features. He had good power but had hypotonia, hyperlaxity, and hyperreflexia in all 4 limbs. MRI showed bilateral symmetric anterior putamen and caudate abnormal signal intensity, indicating caudate atrophy, and symmetric abnormal signal intensity in the basal ganglia. EMG was unremarkable, while whole-exome sequencing test showed homozygous mutation c.91A>T p. (Arg31*) in exon 2 (of 17) of the SERAC1 gene on chromosome 16. Because it was confirmed that mutations in SERAC1 cause MEGDEL syndrome, this explained the patient's signs and symptoms (Fig. 1, 2).

Currently, he has occasional mild choreoathetotic movement with no history of seizure. EEG revealed symmetrical background of $9 \mathrm{~Hz}$ with no epileptiform discharges. Furthermore, he recently started to self-mutilate himself by biting his arms, hands, and fingers till bleeding (Fig. 2). He does not sleep >2-4 h per day, slightly improved on melatonin for 2 weeks but became resistant. Currently on bedtime clonidine with fair response, swallowing assessment revealed poor swallowing coordination and requires gastric tube insertion. Over the last few years, transaminases were fluctuating with highest level were 3 times the normal fold. Lipid profile maintained within normal range. The main treatment of MEGDEL syndrome is supportive and multidisciplinary, involving pediatric neurologists, metabolic pediatricians, dieticians, and physical therapists.

\section{Discussion}

MEGDEL 3-methylglutaconic (MG) aciduria, deafness, encephalopathy, Leigh-like syndrome has an autosomal recessive pattern of inheritance. 3-MG aciduria is a group of different metabolic disorders biochemically confirmed by increased excretion of 3-MG acid. The precise etiology of the increased 3-MG acid excretion in these subtypes has yet to be elucidated.

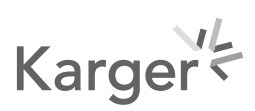




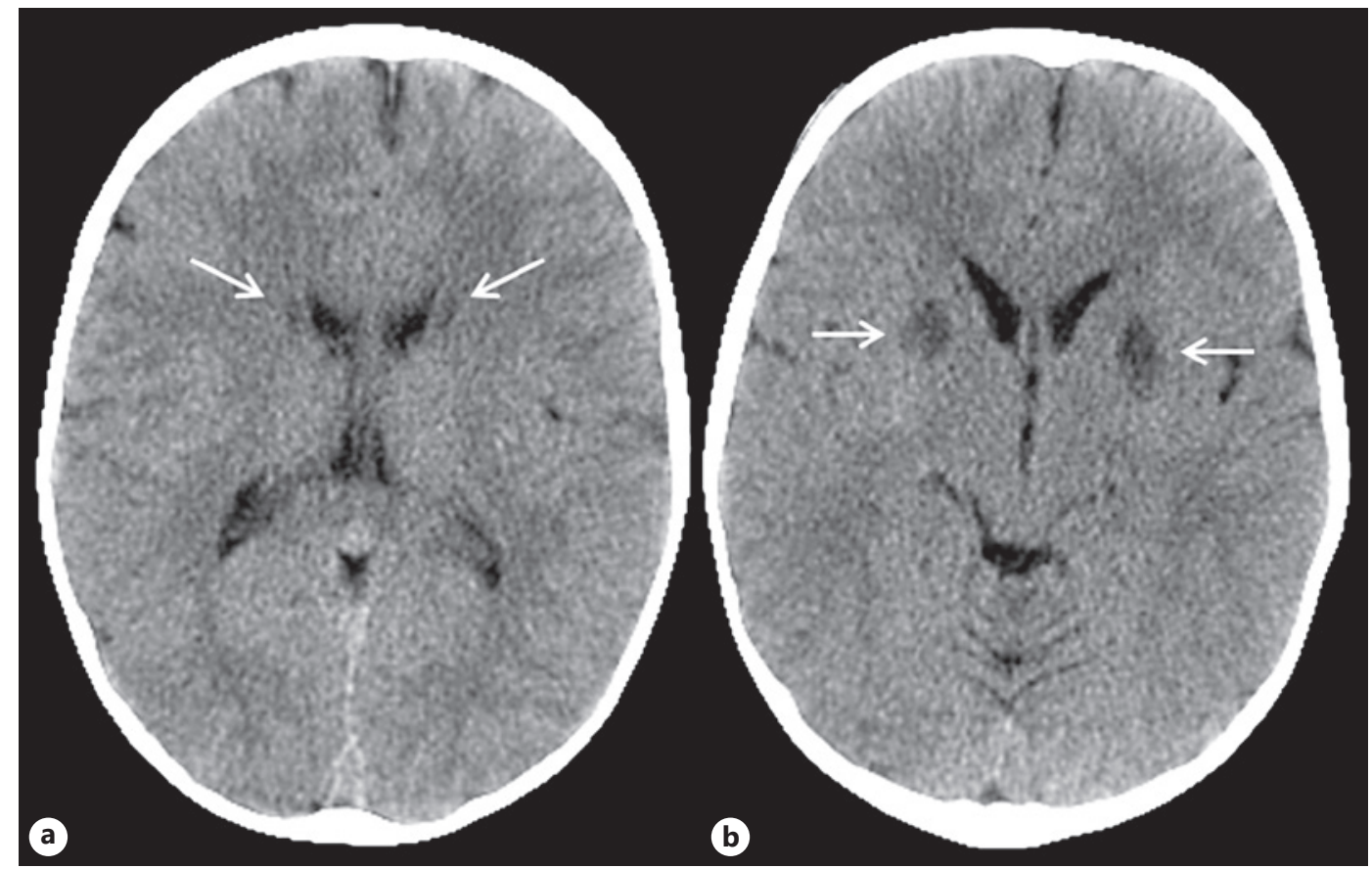

Fig. 1. Axial plain brain CT images show bilateral well-defined hypodensities along caudate nuclei (a) and putamina (b).

Fig. 2. Pictures of both arms revealed frequent different ages ulcers secondary to self-biting (self-mutilation).

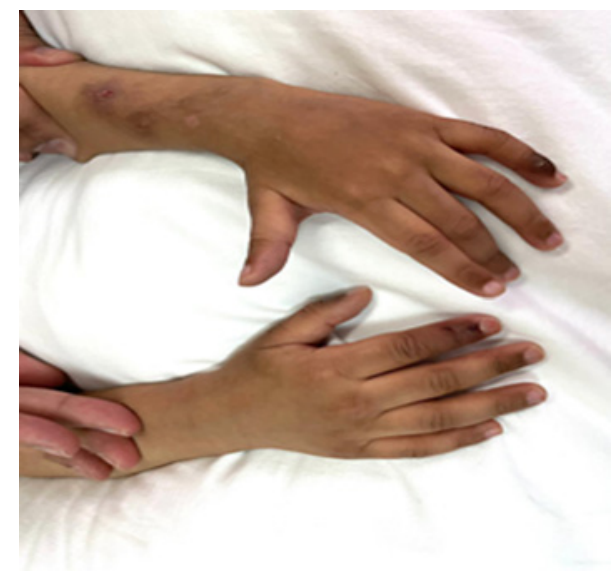

MEGDEL syndrome is considered as a challenging condition because of its rarity [1]. The prevalence and incidence of MEGDEL syndrome remain unknown. Leigh disease and MEGDEL syndrome have superficially similar brain imaging characteristics, making diagnosis even more challenging. Leigh disease is characterized by symmetrical hyperdense lesions in the basal ganglia or brain stem on MRI.

The genetic basis of MEGDEL syndrome was discovered in 2012 when 4 children presented with sensorineural deafness, hypoglycemia, and recurrent lactic acidemia in their first week of life, along with neonatal infections. Muscle biopsies were performed, and biochemical evaluation confirmed the presence of an oxidative phosphorylation deficiency [2]. Here was another report of a child with intrauterine growth retardation who presented with hypoglycemia in the first 2 days of life. The patient had sensorineural deafness by the 
age of 18 months and progressive hypotonia and psychomotor delay. Using exome sequencing, SERAC1 mutation was confirmed. However, by the age of 2 years, the patient had died due to acute pneumonia leading to cardiorespiratory arrest [3]. Furthermore, SERAC1 mutation was also confirmed in 2 siblings with dysmorphic features who were reported to have microcephaly, growth retardation, severe sensorineural deafness, and seizures [4].

In 2017, a multicenter retrospective study was carried out in 67 cases of progressive deafness and dystonia. A total of 41 different SERAC1 variants were identified [5]. SERAC1 mutation is responsible for MEGDEL syndrome [3]. Patients present with mild hypotonia, hyperlaxity, hyperreflexia, and psychomotor delay. The brain MRIs of these patients show symmetric abnormal signal intensity involving the anterior putamen to its posterior tip; the caudate head, body, and tail show significant atrophy. Whole-exome sequencing test is used to confirm the diagnosis of MEGDEL syndrome [6]. His case is complex because of the difficulty in diagnosis and treatment of the syndrome.

A different MEGDEL syndromic presentation was reported in a 7-year-old girl who had severe lactic acidosis and renal failure. The initial evaluation revealed massive pyuria and MGA. She developed progressive dystonia and hypotonia with extrapyramidal symptoms; MRI revealed basal ganglia lesions, and sensorineural deafness was documented when she was 3 years old. MEGDEL syndrome was later confirmed by the presence of a SERAC1 mutation [6].

The typical presentation of MEGDEL syndrome involves major motor symptoms (e.g., dystonia and spasticity) and intellectual disability with deafness, starting at an early age. Late-onset MEGDEL syndrome was reported in a 31-year-old male with mild psychomotor delay. He presented with generalized dystonia and progressive lower limb spasticity triggered by fever. The mitochondrial disease was identified by SERAC1 mutation [7]. Significant milestone delays are found in MEGDEL syndrome. A 5-year-old boy was reported to have a significant history of developmental regression at the age of 3 years, after which he could not walk or pick up objects and demonstrated limited crying and movement. SERAC1 mutation was detected, and MEGDEL syndrome was confirmed [8]. Two cases were reported in India with a history of intellectual disability, dystonia, aciduria, and Leigh-like lesions on brain MRI. SERAC1 mutation confirmed the diagnosis of MEGDEL syndrome [9].

\section{Conclusion}

MEGDEL syndrome is a rare progressive devastating genetic disease with no curative treatment. This case had severe psychomotor regression, self-mutilation, and severe insomnia. More research at the genetic level is needed in the future to reach a clear hypothesis about the disease and its management.

\section{Statement of Ethics}

Written informed consent was obtained from the father of child for publication of this case report and any accompanying images.

\section{Conflict of Interest Statement}

The authors have no conflicts of interest to declare

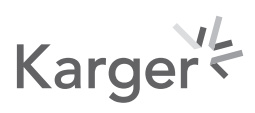




\section{Funding Sources}

The authors did not receive any funding.

\section{Author Contributions}

Salma A. Alshammari: Writing the first draft. Shaikhah Aldossary: Patient care and coordination. Rami Alhazmi: Neuroimaging report and review. Fouad A. Alghamdi: Write and review the draft.

\section{References}

1 Wortmann SB, Van Hasselt PM, Barić I, Burlina A, Darin N, Hörster F, et al. Eyes on MEGDEL: distinctive basal ganglia involvement in dystonia deafness syndrome. Neuropediatrics. 2015;46(2):98-103.

2 Wortmann SB, Vaz FM, Gardeitchik T, Vissers LE, Renkema GH, Schuurs-Hoeijmakers JH, et al. Mutations in the phospholipid remodeling gene SERAC1 impair mitochondrial function and intracellular cholesterol trafficking and cause dystonia and deafness. Nat Genet. 2012;44(7):797-802.

3 Dweikat IM, Abdelrazeq S, Ayesh S, Jundi T. MEGDEL syndrome in a child from Palestine: report of a novel mutation in SERAC1 gene. J Child Neurol. 2015;30(8):1053-6.

4 Giron C, Roze E, Degos B, Méneret A, Jardel C, Lannuzel A, et al. Adult-onset generalized dystonia as the main manifestation of MEGDEL syndrome. Tremor Other Hyperkinet Mov. 2018;8:554.

5 Ünal Ö, Köksal Özgül R, Yücel D, Yalnızoğlu D, Tokatlı A, Serap Sivri H, et al. Two Turkish siblings with MEGDEL syndrome due to novel SERAC1 gene mutation. Turk J Pediatr. 2015;57(4):388-93.

6 Maas RR, Iwanicka-Pronicka K, Kalkan Ucar S, Alhaddad B, AlSayed M, Al-Owain MA, et al. Progressive deafness-dystonia due to SERAC1 mutations: a study of 67 cases. Ann Neurol. 2017;82(6):1004-15.

7 Harbulot C, Paquay S, Dorboz I, Pichard S, Bourillon A, Benoist JF, et al. Transient neonatal renal failure and massive polyuria in MEGDEL syndrome. Mol Genet Metab Rep. 2016;7:8-10.

8 Lumish HS, Yang Y, Xia F, Wilson A, Chung WK. The expanding MEGDEL phenotype: optic nerve atrophy, microcephaly, and myoclonic epilepsy in a child with SERAC1 mutations. JIMD Rep. 2014;16:75-9.

9 Radha Rama Devi A, Lingappa L. Novel mutations in SERAC1 gene in two Indian patients presenting with dystonia and intellectual disability. Eur J Med Genet. 2018;61(2):100-3. 